\title{
Viable entanglement detection of unknown mixed states in low dimensions
}

\author{
Thiago O. Maciel ${ }^{1, *}$ and Reinaldo O. Vianna ${ }^{1, \dagger}$ \\ ${ }^{1}$ Departamento de Física - ICEx - Universidade Federal de Minas Gerais, \\ Av. Antônio Carlos 6627 - Belo Horizonte - MG - Brazil - 31270-901.
}

(Dated: November 11, 2018)

\begin{abstract}
We explore procedures to detect entanglement of unknown mixed states, which can be experimentally viable. The heart of the method is a hierarchy of simple feasibility problems, which provides sufficient conditions to entanglement. Our numerical investigations indicate that the entanglement is detected with a cost which is much lower than full state tomography. The procedure is applicable to both free and bound entanglement, and involves only single copy measurements.

PACS numbers: 03.67.-a
\end{abstract}

\section{INTRODUCTION}

Entanglement is in the heart of Quantum Information, and allows for promising spectacular applications like Quantum Computation and Unconditionally Secure Quantum Cryptography. Our understanding of entanglement has largely grown in the last few years, but the experimental detection is still a daunting challenge. A recent and thorough review of the subject is provided by the Horodecki family [1].

Theoretically, the tool of choice to detect entanglement is an Entanglement Witness (EW) [2]. It consists of a Hermitian operator $(W)$ with non-negative expectation values for all the separable states, but which can have a negative expectation value for an entangled state, in this case, the state is said to be detected by the EW. When it comes to experiments, EWs are not that good, for each state has its own optimal witness. The construction of the optimal EW depends on the knowledge of the state (see [3], for example). There does not exist an EW which detects all the entangled density operators acting on a given Hilbert space. Nevertheless, an EW can detect many states on a certain region of the state space, though it will be optimal just for a restricted family of states. Therefore, when some information about the state is known, an EW can be implemented. For examples of experimental implementations of EWs, consult the references in [1].

Exploring collective measurements to estimate nonlinear functionals of quantum states, Walborn et al. [4] have experimentally measured the concurrence of unknown pure two qubit states, using two copies of the objective state. It has also been extended to the estimation of the concurrence of mixed states, and implemented experimentally [5]. In the case of rank- 2 two-qubit states, it is also possible to measure the concurrence exactly by means of collective measurements on four copies [6].

Here we investigate measurements on single copies of unknown mixed quantum states. The method we propose

\footnotetext{
*Electronic address: maciel@gmail.com

${ }^{\dagger}$ Electronic address: reinaldo@fisica.ufmg.br
}

is simple, and shows to be effective in low dimensions. We are advocating that using sophisticated mathematical tools to characterize entanglement in a data postprocessing fashion, while keeping the experiment as simple as possible, is efficient. Therefore it is an approach in the opposite direction of works like those in $[4,5]$, for example, where entanglement is directly measured in a very elaborate experiment. We have performed numerical tests in systems of two qubits, one qubit and one qutrit and two qutrits. In the case of two qutrits, we have investigated both bound and free entanglement. Though we discuss just bipartite entanglement, the formalism can be straightforwardly applied to the multipartite case. The basic idea is to consider the state written in an orthonormal basis, which can be thought of as a generalized Bloch representation. Then the projections, which are the components of the generalized Bloch vector, are gradually measured. For each set of measurements, it is checked if there is enough information to infer entanglement. In the case of states with Negative Partial Transpose (NPT), we check if there is no state with Positive Partial Transpose (PPT) compatible with the measurements. In the general case, including entangled PPT states [7], we build an entanglement witness compatible with the measurements, using the method described in [3]. In the spirit of data post-processing, we mention the recent techniques independently introduced by Eisert et al. [8] and Gühne et al. [9], that yield bounds to certain entanglement quantifiers, based on the measurement of non-optimal EWs. We note also that Badziag et al. [10] and Hassan et al. [11] have introduced interesting entanglement criteria based on the norm of the generalized Bloch vector. In the context of Quantum Key Distribution, Curty et al. [12] have introduced a method to check the presence of entanglement by means of EWs built with previous measured data.

In the next section, we introduce and illustrate our method. Section III discusses a possible choice of informationanlly complete set of observables, and illustrates how our method would perform with projective measurements. In particular, that section makes clear that, compared to two qubits and two qutrits, it is not obvious how to choose the minimal amount of (or optimal) measurements in a $2 \otimes 3$ system. Section IV offers more ques- 
tions than answers. There we analyze the limitations of the method, studying three representative states, namely, one highly entangled and tow very low entangled states. As presented in this work, our method offers a yes/no answer about the entanglement of a unknown mixed state, but the calculations of section IV suggest that a further development of it could yield a good quantitative estimator of entanglement. Section V concludes the paper.

\section{THEORY}

Given a state represented by the density operator $\rho$, we want to check if it is entangled, without performing a full tomography. As matter of fact, we want to make the least possible number of assumptions about the state. We will present a strategy based on acquisition of partial information about the state, followed by data (post-)processing in form of Semi-Definite Programs (SDP). SDPs can be efficiently solved [13], and have exact solutions. As the numerical tests will show, it is effective in low dimensions. We focus on bipartite states in order to simplify the discussion, but the generalization of the formalism to multipartite states is straightforward.

A state $\rho$, acting on the Hilbert space $\mathcal{H}_{d}=\mathcal{C}^{d_{a}} \times \mathcal{C}^{d_{b}}$, where $d=d_{a} \times d_{b}$, can be written as:

$$
\rho=\sum_{i=0}^{d_{a}^{2}-1} \sum_{j=0}^{d_{b}^{2}-1} r_{i j} P_{i j},
$$

where the $P_{i j}$ are observables forming a complete basis in the Hilbert-Schmidt space, and $r_{i j}=\operatorname{Tr}\left(\rho P_{i j}\right) \in \mathcal{R}$.

One possible choice for these observables is $P_{i j}=$ $\sigma_{i}^{d_{a}} \otimes \sigma_{j}^{d_{b}}$, with the $\sigma_{i}^{d_{s}}$ being $S U\left(d_{s}\right)$ matrices, i.e., generalizations of the Pauli matrices, where $\sigma_{0}^{d_{s}}$ stands for the identity matrix, and $r_{00}=1 / d$. In this case, the state can also be written with explicit local and non-local parts, and we have an expression that can be thought of as a generalized Bloch representation, namely,

$$
\begin{array}{r}
\rho=\frac{1}{d}\left(\mathbf{I}_{\mathbf{d}_{\mathbf{a}}} \otimes \mathbf{I}_{\mathbf{d}_{\mathbf{b}}}+\vec{r}_{a} \cdot \vec{\sigma}^{d_{a}} \otimes\right. \\
\mathbf{I}_{\mathbf{d}_{\mathbf{b}}}+\mathbf{I}_{\mathbf{d}_{\mathbf{a}}} \otimes \vec{r}_{b} \cdot \vec{\sigma}^{d_{b}}+ \\
\left.\sum_{i=1}^{d_{a}^{2}-1} \sum_{j=1}^{d_{b}^{2}-1} t_{i j} \sigma_{i}^{d_{a}} \otimes \sigma_{j}^{d_{b}}\right),
\end{array}
$$

where $\mathbf{I}_{\mathbf{d}_{\mathbf{s}}}$ is the $d_{s} \times d_{s}$ identity matrix, $\vec{\sigma}^{d_{s}}$ are the matrices for $S U\left(d_{s}\right), \vec{r}_{s} \in \mathcal{R}^{d_{s}^{2}-1}$, and finally $t_{i j} \in \mathcal{R}$. Note that $\vec{r}_{a}$ and $\vec{r}_{b}$ are the local parameters, defining the reduced density matrices, namely:

$$
\rho_{a} \equiv \operatorname{Tr}_{b} \rho=\frac{1}{d_{a}}\left(\mathbf{I}_{\mathbf{a}}+\overrightarrow{\mathbf{r}} \cdot \vec{\sigma}^{\mathbf{d}_{\mathbf{a}}}\right),
$$

where $T r_{b}$ is the partial trace on subsystem $b$, and an analogous expression for $\rho_{b}$. The non-local parameters,

$$
t_{i j}=\operatorname{Tr}\left(\rho \sigma_{i}^{d_{a}} \otimes \sigma_{j}^{d_{b}}\right)=\left\langle T_{i j}\right\rangle
$$

form a real matrix $T$, and are responsible for the classical and quantum correlations in $\rho$. Note that the parameters in Eq. 1 or Eq. 2 must be real in order to $\rho$ to be Hermitian, but it does not guarantee its positivity.

We will introduce some procedures to check the entanglement of an unknown state, based on partial information about it. This partial information consists of the knowledge of some of the $r_{i j}$ (viz. Eq. 1), eventually enriched with some further characteristic of the state, as the fact that it is NPT, or its marginals are known (viz. Eq. 3).

As it is well known, it is harder to check the entanglement of a bound entangled state than a free entangled one. From the theoretical point of view, it is easy to know if the latter are entangled, for they have negative partial transpose, which is known as the Peres-Horodecki criterion $[2,14]$. But if the state is PPT, we need an entanglement witness. The known examples of bound entangled states show very low entanglement, therefore they will be more difficult to be checked experimentally.

Now we present our first procedure, which checks entanglement in NPT bipartite states. The method we propose can be thought of as a way of checking the PeresHorodecki criterion. Assuming the knowledge of $n(n \leq$ $\left.d^{2}-1\right)$ of the parameters $r_{k} \equiv r_{i j}\left(k=1,2, \ldots, d^{2}-1\right)$ in Eq. 1, we check the existence of a PPT state compatible with the available information. The nonexistence of such a state witnesses the entanglement of the state of interest. This can be done by means of the following very simple SDP:

$$
\begin{gathered}
\text { determine } \varrho \\
\text { subject to }\left\{\begin{array}{l}
\varrho \geq 0 \\
\operatorname{Tr}(\varrho)=1 \\
\varrho \Gamma 0 \\
\operatorname{Tr}\left(\varrho P_{k}\right)=r_{k}, \quad k=1,2, \ldots, n .
\end{array}\right.
\end{gathered}
$$

This SDP is a feasibility program. $\varrho^{\Gamma}$ stands for the partial transpose of $\varrho$. When this program is infeasible, i.e., when there is no PPT state compatible with the available data, we are certain that the unknown $\rho$ is NPT and, therefore, entangled.

It could happen that the state of interest has passed through some known decoherence channel, which restricts the state's marginals to some known form. One example is the Werner states [15], which correspond to depolarized states whose marginals are maximally mixed. The program in Eq. 5 can be easily modified to include this additional information, which corresponds to further constraints in the SDP, namely:

$$
\begin{aligned}
& \text { determine } \varrho \\
& \text { subject to }\left\{\begin{array}{l}
\varrho \geq 0 \\
\operatorname{Tr}(\varrho)=1 \\
\varrho \Gamma \geq 0 \\
\varrho_{a}=\rho_{a} \\
\varrho_{b}=\rho_{b} \\
\operatorname{Tr}\left(\varrho P_{k}\right)=r_{k}, \quad k=1,2, \ldots, n .
\end{array}\right.
\end{aligned}
$$


Programs in Eq. 5 and in Eq. 6 determine the projection of the state of interest in a certain hyperplane (in the Hilbert-Schimidt space), and check the existence of the family of PPT states with the same projection. If there is no such state, it means that the measured state is NPT, and therefore entangled. This suffices to check entanglement in spaces $2 \times 2$ (qubit-qubit) and $2 \times 3$ (qubit-qutrit) [2]. In larger spaces, this approach still works for the NPT states, but it will not detect entangled PPT states. Now we introduce a procedure that, in principle, can detect both free and bound entangled states.

When the state of interest is in a space which allows for bound entanglement [7], we need an entanglement witness to check if it is separable or not. If we eliminate the constraint of positivity of the partial transpose in the programs of Eqs. 5 and 6 , namely, $\varrho^{\Gamma} \geq 0$, those programs return a state $\varrho$, which can be PPT or not, compatible with the available data. We then build an optimal entanglement witness $\left(W_{\varrho}\right)$ to $\varrho$, and use it to estimate the entanglement of $\rho$, i.e., $\operatorname{Tr}\left(W_{\varrho} \rho\right) \sim \operatorname{Tr}\left(W_{\varrho} \varrho\right)$. Remember that an EW is a Hermitian operator with non-negative expectation values on separable states, but which can have a negative expectation value on an entangled state, in this case, we say that the EW detects the entangled state. The optimal EW of a state yields the most negative expectation value, when compared to any other EW of the same kind, therefore $\operatorname{Tr}\left(W_{\varrho} \rho\right)>\operatorname{Tr}\left(W_{\rho} \rho\right)$. Note that EWs can be chosen to correspond to different entanglement quantifiers [16]. The EWs in this work have the constraint $\operatorname{Tr}(W)=1$, and correspond to the random robustness $[17,18]$, which measures how resilient to white noise is the entanglement.

We need an error bar to our entanglement estimate given by $\operatorname{Tr}\left(W_{\varrho} \varrho\right)$. In order to do that, we rewrite Eq. 1 as:

$$
\varrho=\sum_{k=1}^{n} r_{k} P_{k}+\sum_{j=n+1}^{d^{2}} r_{j} P_{j} .
$$

The first summation corresponds to the known data. Let's call it $\varrho_{\text {known }}$. Of course, $\rho_{\text {known }}=\varrho_{\text {known }}$. The second summation is yielded either by the program in Eq. 5) or Eq. 6, and we call it @unknown. Now we can write our entanglement estimate as:

$$
\operatorname{Tr}\left(W_{\varrho} \rho\right)=\operatorname{Tr}\left(W_{\varrho} \varrho\right) \pm\left|\operatorname{Tr}\left(W_{\varrho} \varrho_{\text {unknown }}\right)\right| .
$$

The techniques we use to build the optimal EW are based on SDPs, and are described in [3].

In Fig. 1, we show how the method performs for two qubits, one qubit and one qutrit, and two qutrits. Each graph is built out of $10^{4}$ random NPT entangled states. We plot the efficacy of entanglement detection (i.e., number of states detected as entangled divided by $10^{4}$ ) in the sample of states, against the number of measured non-local parameters (viz. Eq. 4). Every time the program in Eq. 5 or in Eq. 6 is infeasible for a given state, it means that the measured data were sufficient to detect entanglement. In the case of two qutrits, we also test the EW approach of Eq. 8. A state is considered successfully detected as entangled, when both $\operatorname{Tr}\left(W_{\varrho} \varrho\right)+\left|\operatorname{Tr}\left(W_{\varrho} \varrho_{\text {unknown }}\right)\right|$ and $\operatorname{Tr}\left(W_{\varrho} \varrho\right)-\left|\operatorname{Tr}\left(W_{\varrho} \varrho_{\text {unknown }}\right)\right|$ are negative. About $70 \%$ of the states are detected as entangled, with an effort which is roughly half of a full state tomography. On one hand, the less entangled is the state, more information we need to infer its entanglement. Therefore, the graphs show $100 \%$ efficacy only when all the tomographic parameters are measured. On the other hand, highly entangled states are detected with the knowledge of only a few non-local parameters.

\section{CHOOSING WHAT TO MEASURE IN THE LABORATORY}

In the last section, we have described the general idea behind detecting entanglement of unknown states, based on partial information. Our main goal was to show that the proposed data post-processing is effective. Now we want to discuss how our technique could be actually implemented with projective measurements. A sensible way to do this is by grouping the observables in the smallest number of maximal commuting classes. Commuting observables share a common set of eigenvectors and, consequently, can be simultaneously measured (i.e., can be simultaneously diagonalized). Therefore, such a scheme would yield the smallest number of complete projective measurements to be done.

Let's fix the basis of observables. For a Hilbert space of dimension $d_{s}$, we introduce the shift and clock operators, namely:

$$
X \equiv \sum_{j=0}^{d_{s}-1}|j+1\rangle\langle j|
$$

and

$$
Z \equiv \sum_{j=0}^{d_{s}-1} \exp \left(\frac{2 \pi i j}{2}\right)|j\rangle\langle j|,
$$

where $\left\{|j\rangle, j=0, \ldots, d_{s}-1\right\}$ is an orthonormal basis. For dimension 2, these operators are the usual Pauli matrices. We also define:

$$
Y \equiv X Z
$$

and, for $d>2$,

$$
V \equiv X Z^{2}
$$

Two-particle observables are now defined as tensor products of powers of these operators. For two qubits, one qubit and one qutrit, and two qutrits, Table I shows the complete bases of observables, with a convenient labelling [19]. 

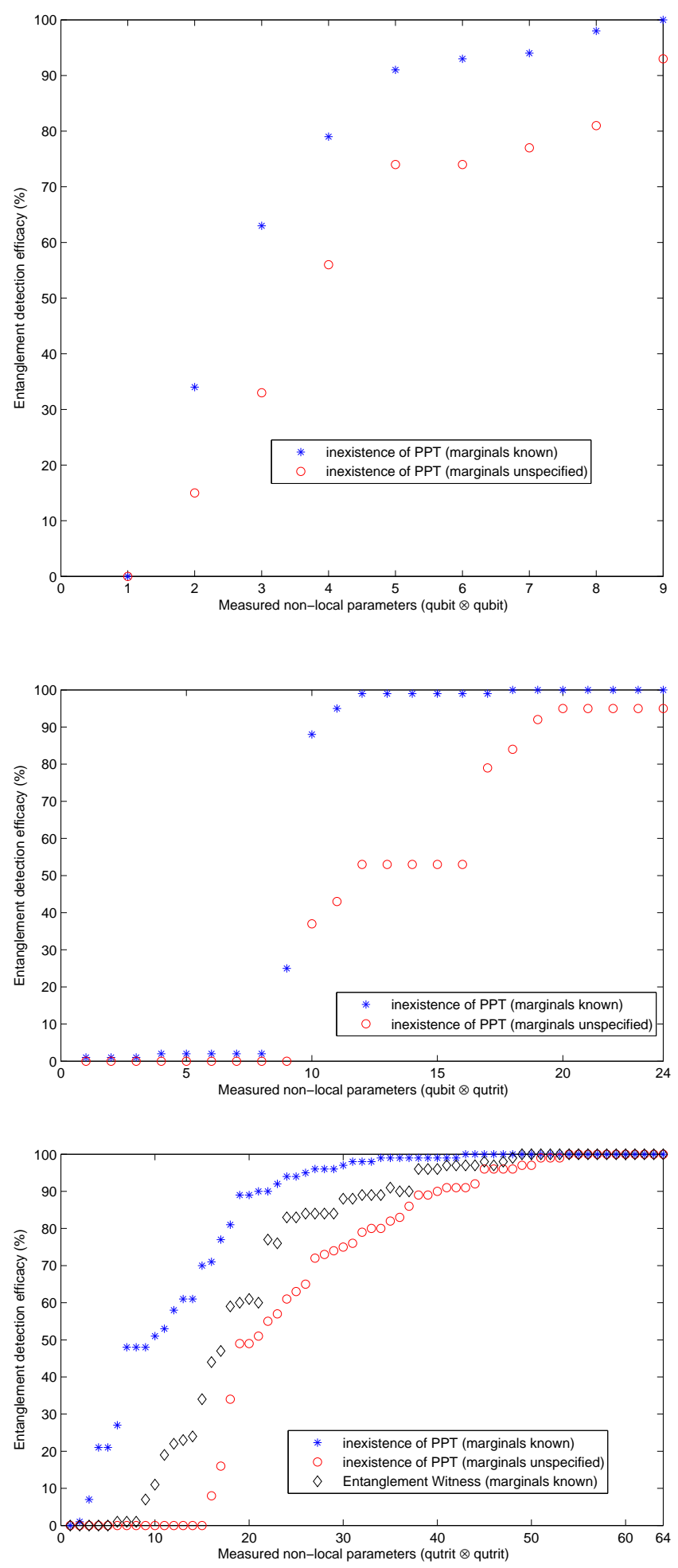

FIG. 1: Fraction of success of entanglement detection against the number of measured non-local parameters (viz. Eq. 4), for a sample of $10^{4}$ random NPT states, using the approaches described in Eqs. 5 and 6, for two qubits, one qubit and one qutrit, and two qutrits. For two qutrits, we also show the results using the EW (Eq. 8).

\begin{tabular}{c||c|c|c|c}
$2 \otimes 2$ & $\mathrm{I}$ & $\mathrm{Z}$ & $\mathrm{X}$ & $\mathrm{Y}$ \\
\hline \hline $\mathrm{I}$ & 0 & 13 & 14 & 15 \\
\hline $\mathrm{Z}$ & 1 & 4 & 7 & 10 \\
\hline $\mathrm{X}$ & 2 & 5 & 8 & 11 \\
\hline $\mathrm{Y}$ & 3 & 6 & 9 & 12 \\
\hline \hline $2 \otimes 3$ & $\mathrm{I}$ & $\mathrm{Z}$ & $\mathrm{X}$ & $\mathrm{Y}$ \\
\hline \hline $\mathrm{I}$ & 0 & 33 & 34 & 35 \\
\hline $\mathrm{Z}$ & 1 & 9 & 17 & 25 \\
\hline $\mathrm{X}$ & 2 & 10 & 18 & 26 \\
\hline $\mathrm{Y}$ & 3 & 11 & 19 & 27 \\
\hline $\mathrm{V}$ & 4 & 12 & 20 & 28 \\
\hline$Z^{2}$ & 5 & 13 & 21 & 29 \\
\hline$X^{2}$ & 6 & 14 & 22 & 30 \\
\hline$Y^{2}$ & 7 & 15 & 23 & 31 \\
\hline$V^{2}$ & 8 & 16 & 24 & 32 \\
\hline \hline $\mathrm{Z}$ & \multicolumn{1}{|c|}{} & \multicolumn{2}{|c}{}
\end{tabular}

\begin{tabular}{c||c|c|c|c|c|c|c|c|c|c}
$3 \otimes 3$ & $\mathrm{I}$ & $\mathrm{Z}$ & $\mathrm{X}$ & $\mathrm{Y}$ & $\mathrm{V}$ & $Z^{2}$ & $X^{2}$ & $Y^{2}$ & $V^{2}$ \\
\hline \hline $\mathrm{I}$ & 0 & 73 & 74 & 75 & 76 & 77 & 78 & 79 & 80 \\
\hline $\mathrm{Z}$ & 1 & 9 & 17 & 25 & 33 & 41 & 49 & 57 & 65 \\
\hline $\mathrm{X}$ & 2 & 10 & 18 & 26 & 34 & 42 & 50 & 58 & 66 \\
\hline $\mathrm{Y}$ & 3 & 11 & 19 & 27 & 35 & 43 & 51 & 59 & 67 \\
\hline $\mathrm{V}$ & 4 & 12 & 20 & 28 & 36 & 44 & 52 & 60 & 68 \\
\hline$Z^{2}$ & 5 & 13 & 21 & 29 & 37 & 45 & 53 & 61 & 69 \\
\hline$X^{2}$ & 6 & 14 & 22 & 30 & 38 & 46 & 54 & 62 & 70 \\
\hline$Y^{2}$ & 7 & 15 & 23 & 31 & 39 & 47 & 55 & 63 & 71 \\
\hline$V^{2}$ & 8 & 16 & 24 & 32 & 40 & 48 & 56 & 64 & 72 \\
\hline
\end{tabular}

TABLE I: Complete bases of observables in Hilbert spaces of dimensions $2 \otimes 2,2 \otimes 3$ and $3 \otimes 3$. Each two-particle observable is the tensor product between one operator of the first line by one operator of the first column.

Now we can group the observables of Table I in maximally commuting classes. For two qubits, we have the 5 classes [20] shown in Table II; for one qubit and one qutrit, there are 12 classes, as shown in Table III; and finally, for two qutrits, there are the 10 classes [21, 22] shown in Table IV. Note that, for two qubits and two qutrits, the classes are disjoint sets. In each case, the simultaneous eigenvectors of each class form a set of $\mathrm{Mu}-$ tually Unbiased Bases (MUB) [23, 24], in the sense that any two vectors of different bases have the same overlap's absolute value. In the case of the $2 \otimes 3$ system, the classes are neither disjoint and nor minimal. With 5 observables in each class, the minimal number of classes, for a total of 35 distinct operators, should be 7 . It is the case for the systems $2 \otimes 2$ and $3 \otimes 3$, with 15 observables divided in 5 classes of 3 operators, and 80 observables divided in 10 classes of 8 operators, respectively. It is conjectured that there is no informationally complete set (in the tomographic sense) of MUBs for the $2 \otimes 3$ system [25-27], and it is known that generalized Pauli matrices (which is our choice of observables) are not extensible to MUBs [28].

In Fig. 2, we repeat the calculations of section II, namely Eqs. 1, 5 and 8, but now using the MUB projectors for the two qubits and two qutrits.

Though we do not know MUBs for the $2 \otimes 3$ system, 


$$
\begin{aligned}
& C_{1}=\left\{\begin{array}{lll}
1 & 4 & 13
\end{array}\right\} \\
& C_{2}=\left\{\begin{array}{lll}
2 & 8 & 14
\end{array}\right\} \\
& C_{3}=\left\{\begin{array}{lll}
3 & 12 & 15
\end{array}\right\} \\
& C_{4}=\left\{\begin{array}{lll}
5 & 9 & 10
\end{array}\right\} \\
& C_{5}=\left\{\begin{array}{lll}
6 & 7 & 11
\end{array}\right\}
\end{aligned}
$$

TABLE II: Five maximally commuting classes of observables (viz. Table I) for two qubits. The common eigenvectors of each class form a set of MUBs.

$$
\begin{aligned}
C_{1} & =\left\{\begin{array}{lllll}
1 & 5 & 33 & 9 & 13
\end{array}\right\} \\
C_{2} & =\left\{\begin{array}{llllll}
2 & 6 & 33 & 10 & 14
\end{array}\right\} \\
C_{3} & =\left\{\begin{array}{lllll}
3 & 7 & 33 & 11 & 15
\end{array}\right\} \\
C_{4} & =\left\{\begin{array}{lllll}
4 & 8 & 33 & 12 & 16
\end{array}\right\} \\
C_{5} & =\left\{\begin{array}{lllll}
1 & 5 & 34 & 17 & 21
\end{array}\right\} \\
C_{6} & =\left\{\begin{array}{llllll}
2 & 6 & 34 & 18 & 22
\end{array}\right\} \\
C_{7} & =\left\{\begin{array}{llllll}
3 & 7 & 34 & 19 & 23
\end{array}\right\} \\
C_{8} & =\left\{\begin{array}{llllll}
4 & 8 & 34 & 20 & 24
\end{array}\right\} \\
C_{9} & =\left\{\begin{array}{llllll}
1 & 5 & 35 & 25 & 29
\end{array}\right\} \\
C_{10} & =\left\{\begin{array}{lllll}
2 & 6 & 35 & 26 & 30
\end{array}\right\} \\
C_{11} & =\left\{\begin{array}{lllll}
3 & 7 & 35 & 27 & 31
\end{array}\right\} \\
C_{12} & =\left\{\begin{array}{lllll}
4 & 8 & 35 & 28 & 32
\end{array}\right\}
\end{aligned}
$$

TABLE III: Twelve maximally commuting classes of observables (viz. Table I) for qubit $\otimes$ qutrit.

we still want to do the minimal number of projective measurements in the laboratory. To make a complete tomography, we need a set of 35 informationally complete projectors. Measuring in the basis of common eigenvectors of each of the 12 classes (Table III), the numbers of independent projective measurements extracted from each class are, respectively, $5,4,4,4,3,2,2,2,3,2,2$, 2 . These 35 projectors, which are linearly independent in the Hilbert-Schimidt space, can be sorted in 7 sets of 5 , and re-orthonormalized in order to correspond to 7 complete projective measurements $(7$ observables $)$. The results of measurements in these two different bases are shown in Fig. 3.

$$
\begin{aligned}
C_{1} & =\left\{\begin{array}{llllllll}
1 & 5 & 73 & 9 & 13 & 77 & 41 & 45
\end{array}\right\} \\
C_{2} & =\left\{\begin{array}{lllllllll}
2 & 6 & 74 & 18 & 22 & 78 & 50 & 54
\end{array}\right\} \\
C_{3} & =\left\{\begin{array}{llllllll}
3 & 7 & 75 & 27 & 31 & 79 & 59 & 63
\end{array}\right\} \\
C_{4} & =\left\{\begin{array}{llllllll}
4 & 8 & 76 & 36 & 40 & 80 & 68 & 72
\end{array}\right\} \\
C_{5} & =\left\{\begin{array}{llllllll}
10 & 46 & 33 & 19 & 32 & 69 & 60 & 55
\end{array}\right\} \\
C_{6} & =\left\{\begin{array}{llllllll}
11 & 47 & 17 & 28 & 38 & 53 & 66 & 64
\end{array}\right\} \\
C_{7} & =\left\{\begin{array}{llllllll}
12 & 48 & 25 & 34 & 23 & 61 & 51 & 70
\end{array}\right\} \\
C_{8} & =\left\{\begin{array}{llllllll}
14 & 42 & 29 & 20 & 39 & 57 & 67 & 56
\end{array}\right\} \\
C_{9} & =\left\{\begin{array}{llllllll}
15 & 43 & 37 & 26 & 24 & 65 & 52 & 62
\end{array}\right\} \\
C_{10} & =\left\{\begin{array}{llllllll}
16 & 44 & 21 & 35 & 30 & 49 & 58 & 71
\end{array}\right\}
\end{aligned}
$$

TABLE IV: Ten maximally commuting classes of observables (viz. Table I) for two qutrits. The common eigenvectors of each class form a set of MUBs.
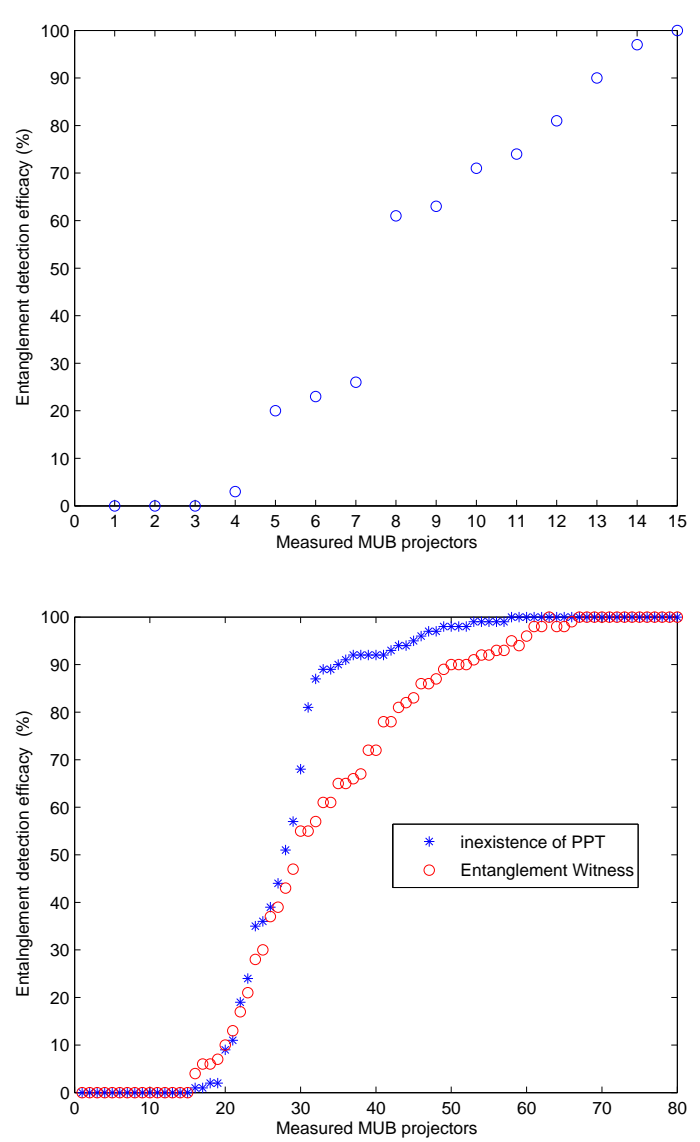

FIG. 2: Fraction of success of entanglement detection against the number of measured MUB projectors (viz. Eq. 1), for a sample of $10^{4}$ random NPT states, using the approaches described in Eq. 5 (Peres-Horodecki criterion), for two qubits (top), and Eqs. 5 and 8 (EW) for two qutrits (bottom).

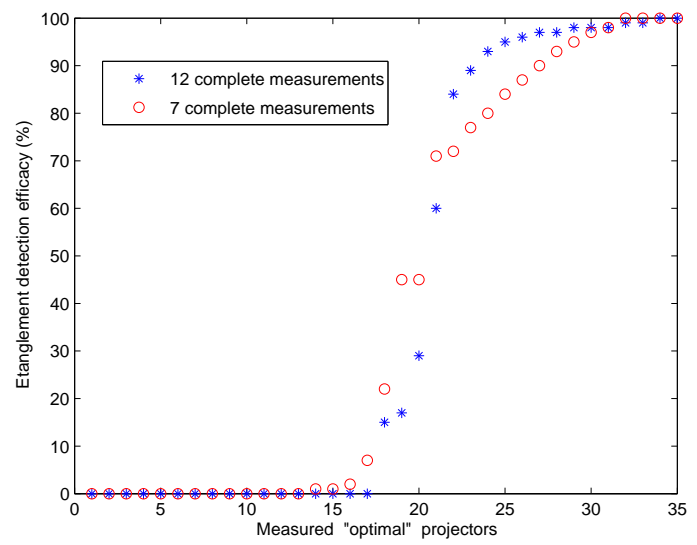

FIG. 3: Fraction of success of entanglement detection against the number of optimal projectors (viz. Eq. 1 and Table III), for a sample of $10^{4}$ random NPT states, using the approach described in Eq. 5 (Peres-Horodecki criterion), for the $2 \otimes 3$ system. 


\section{ESTIMATED EW AND LOW-ENTANGLED STATES}

In sections II and III, we have discussed how we could detect the entanglement of unknown NPT states, based on partial information. In particular, the method we have proposed to check the Peres-Horodecki criterion (Eq. 5) is rigorous, yields an exact answer and, according to our numerical tests, performs nicely for the systems $2 \otimes 2$, $2 \otimes 3$ and $3 \otimes 3$, as shown in Figs. 2 and 3 . On the other hand, our proposed EW estimate (Eq. 8) needs to be better understood. Figs. 1 and 2 are numerical evidence for the correctness of Eq. 8, but we are lacking a rigorous proof for the exact expression of our error bar. In this section, we will study the performance of Eq. 8 in 3 particular states, being one highly entangled two-qutrit Werner state [15] and two very low entangled states, being one of them also a two-qutrit Werner state, and the other one a two-qutrit bound entangled state [29]. It will add further evidence of the correctness of Eq. 8, and will show that our proposed error bar is too big, i.e., it seems that $\operatorname{Tr}\left(W_{\varrho} \varrho\right)$ is a very good upper bound to $\operatorname{Tr}\left(W_{\varrho} \rho\right)$, much better than we expected, and there must be a tighter error bar, but we couldn't devise it yet. Note that $\operatorname{Tr}\left(W_{\varrho} \rho\right)$ is certainly an upper bound to $\operatorname{Tr}\left(W_{\rho} \rho\right)$.

The two-qudit (for our purposes $d=3$ ) Werner states [15] can be written as:

$$
\rho_{W}=\frac{I_{d}+\beta F_{d}}{d^{2}+d \beta}
$$

with $-1 \leq \beta \leq 1 . \rho_{W}$ is separable for $\beta \geq-\frac{1}{d}$. $F_{d}$ is a swap operator for two qudits,

$$
F_{d}=\sum_{i, j=1}^{d}|i j\rangle\langle j i| .
$$

The two-qutrit bound entangled state we use is picked up from the following family of states [29]:

$$
\rho_{H}=\frac{2}{7}\left|\phi_{3}^{+}\right\rangle\left\langle\phi_{3}^{+}\right|+\frac{\lambda}{7} \sigma_{+}+\frac{5-\lambda}{7} \sigma_{-}, 2 \leq \lambda \leq 5,
$$

where

$$
\left|\phi_{3}^{+}\right\rangle\left\langle\phi_{3}^{+}\left|=\frac{1}{\sqrt{3}} \sum_{i, j=0}^{2}\right| i i\right\rangle\langle j j|
$$

is the density matrix for the maximally entangled state, and

$$
\begin{gathered}
\sigma_{+}=\frac{1}{3}(|01\rangle\langle 01|+| 12\rangle\langle 12|+| 20\rangle\langle 20|), \\
\sigma_{-}=\frac{1}{3}(|10\rangle\langle 10|+| 21\rangle\langle 21|+| 02\rangle\langle 02|)
\end{gathered}
$$
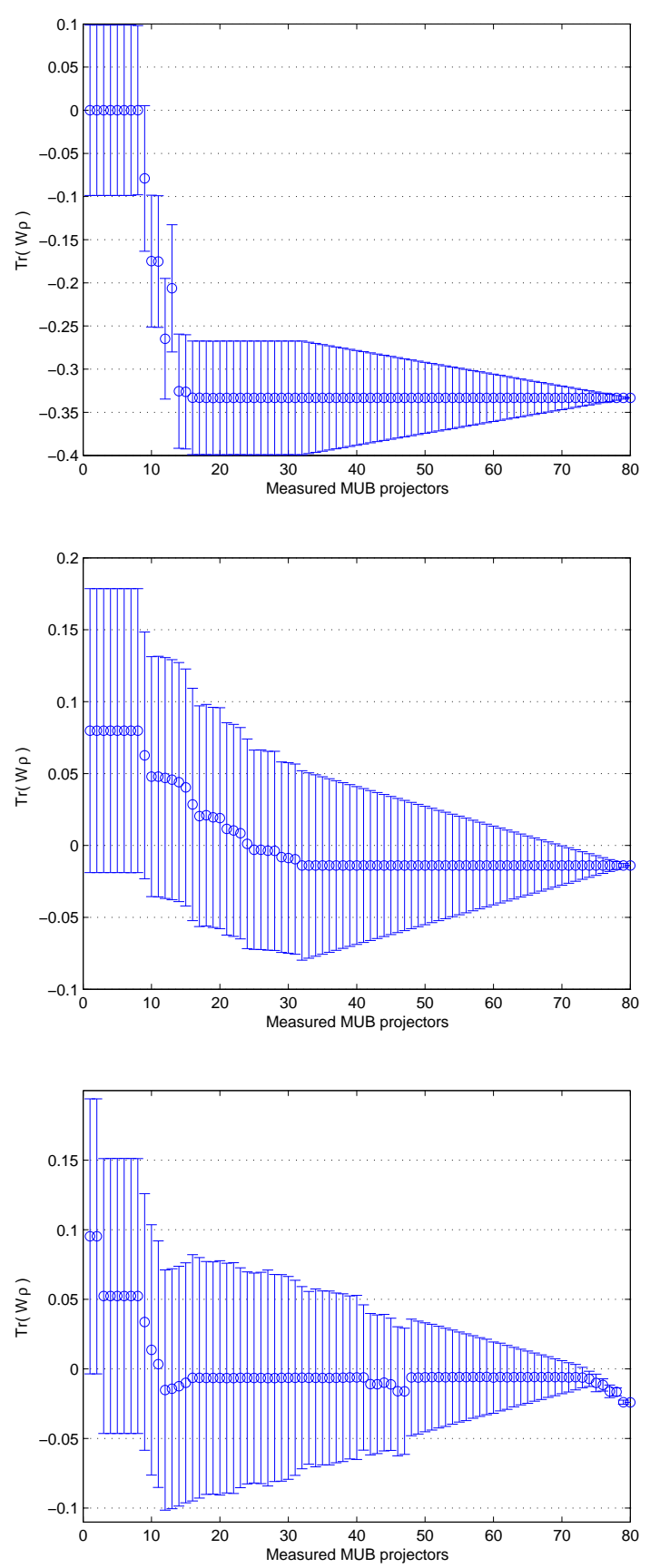

FIG. 4: Estimated EW with its error bar (Eq. 8) for 3 particular two-qutrit states: (top) Werner state with $\beta=-1$, (middle) Werner state with $\beta=-0.37$, (bottom) Horodecki bound entangled state with $\lambda=3.9$. In each case, the exact value for $\operatorname{Tr}\left(W_{\rho} \rho\right)$ corresponds to the mark 80 in the abscissa.

are two separable states. With these definitions, the character of $\rho_{H}$ changes with $\lambda$ according to

$$
\rho_{H}= \begin{cases}\text { separable, } & 2 \leq \lambda \leq 3, \\ \text { bound entangled, } & 3<\lambda \leq 4, \\ \text { free entangled, } & 4<\lambda \leq 5\end{cases}
$$


In Fig. 4, we see the results yielded by Eq. 8 applied to Werner states (Eq. 13) with $\beta=-1\left(\operatorname{Tr}\left(W_{\rho_{W}} \rho_{W}\right)=\right.$ $-1 / 3)$ and $\beta=-0.37\left(\operatorname{Tr}\left(W_{\rho_{W}} \rho_{W}\right)=-0.014\right)$, and to the Horodecki bound entangled state (Eq. 15) with $\lambda=3.9\left(\operatorname{Tr}\left(W_{\rho_{H}} \rho_{H}\right)=-0.024\right)$. As we have mentioned before, a state is considered detected as entangled when the error bar resides entirely in the entangled region. We see that the highly entangled Werner state is detected with just 11 measurements. On the other hand, the two low entangled states are detected after the $70^{t h}$ measurement. The Peres-Horodecki criterion (Eq. 5) detects the low entangled Werner state in the $32^{\text {nd }}$ measurement but, of course, it is not applicable to the PPT state. Ignoring the error bar, note that the estimated EW never superestimated the entanglement; it yielded the exact results for the Werner states after the $17^{\text {th }}$ measurement, for the most entangled state, and after the $32^{\text {nd }}$ measurement, for the low entangled state; and, finally, it detected the bound entangled state after 10 measurements.

\section{CONCLUSION}

We discussed data post-processing strategies to characterize entanglement of unknown mixed states, based on partial knowledge of the state. The method is guaranteed to work, for it converges to a full state tomography. We applied our method in systems of dimension $2 \otimes 2$, $2 \otimes 3$ and $3 \otimes 3$. Our numerical investigations showed that entanglement can be detected with a cost which is much lower than full state tomography, when the entanglement is not very small. For low entangled states, including PPT ones, we presented a method to construct entanglement witnesses (EW). The EWs have an error bar that monotonically diminishes with the increase of information about the state. Our tests suggest that the error bar is too big, for ignoring it, the entanglement estimate yielded by the EW is always a lower bound to the true entanglement. Therefore we believe that a tighter error bar could be calculated, but we weren't able to prove it yet.

We also discussed the choice of observables to be measured in the laboratory. In particular, we noted that the choice is not obvious in the case of the $2 \otimes 3$ system, when one is willing to measure the smallest set of informationally complete projectors. Nevertheless, we offered a method to construct these minimal informationally complete sets, in the case of projective measurements.

The application of our approach to multipartite systems is straightforward, at the level of the formalism. As a matter of fact, we performed some tests on NPT states of three qubits, obtaining results similar to the ones we presented for the bipartite systems.

It would be interesting to investigate how our approach could be adapted to study other properties, as the purity of a state, for example. In this case, quadratic or higher degree non-linear programs would be necessary.

ACKNOWLEDGEMENTS: Financial support provided by the Brazilian agencies FAPEMIG and CNPQ. We thank S. Pádua for the fruitful discussions about measurements. ROV is indebted to G.G. Silva for her overall support.
[1] R. Horodecki, P. Horodecki, M. Horodecki and K. Horodecki, Rev. Mod. Phys. 81, 865 (2009).

[2] M. Horodecki, P. Horodecki and R. Horodecki, Phys. Lett. A 223, 8 (1996).

[3] F.G.S.L Brandão and R.O. Vianna, Phys. Rev. Lett. 93, 220503 (2004).

[4] S.P. Walborn, P.H. Souto Ribeiro, L. Davidovich, F. Mintert, A. Buchleitner, Nature, 440, 1022 (2006). F. Mintert, M. Kus, A. Buchleitner, Phys. Rev. Lett. 95, 260502 (2005).

[5] C. Schmid, N. Kiesel, W. Wieczorek, H. Weinfurter, F. Mintert, A. Buchleitner, Phys. Rev. Lett. 101, 260505 (2008). F. Mintert, A. Buchleitner, Phys. Rev. Lett. 98, 140505 (2007).

[6] C. Yu, C. Li, H. Song, Phys. Rev. A 77, 012305 (2008).

[7] M. Horodecki, P. Horodecki, R. Horodecki, Phys. Rev. Lett. 80, 5239 (1998).

[8] J.Eisert, F.G.S.L. Brandão, K.M.R. Audenaert, New J. Phys 9, 46 (2007).

[9] O. Gühne, M. Reimpell, R.F. Werner, Phys. Rev. Lett. 98, 110502 (2007).

[10] P. Badziag, C. Brukner, W. Laskowski, T. Paterek, M. Zukowski, Phys. Rev. Lett. 100, 140403 (2008).

[11] A.S.M. Hassan, P.S. Joag, Phys. Rev. A 77, 062334 (2008).
[12] M. Curty, M. Lewenstein, N.Lütkenhaus, Phys. Rev. Lett. 92, 217903 (2004).

[13] J.F. Sturm, Optimizations Methods and Software 11-12, 625 (1999), SEDUMI: http://fewcal.kub.nl/sturm/softwares/sedumi.html; J. Löfberg, YALMIP : A Toolbox for Modeling and Optimization in MATLAB, Proceedings of the CACSD Conference, 2004, Taipei, Taiwan, http://control.ee.ethz.ch/ joloef/yalmip.php;

S. Boyd, L. Vandenberghe, Convex Optimization, (Cambridge University Press, Cambridge, 2000).

[14] A. Peres, Phys. Rev. Lett. 77, 1413 (1996).

[15] R.F. Werner, Phys. Rev. A 40, 4277 (1989).

[16] F.G.S.L. Brandão, Phys. Rev. A, 72, 022310 (2005).

[17] G. Vidal and B. Tarrach, Phys. Rev. A 59,141 (1999).

[18] F.G.S.L. Brandão and R.O. Vianna, Int. J. Quantum Inf. 4, 331 (2006).

[19] M. Planat, A.C. Baboin, M. Saniga, Int. J. Theor. Phys 47, 1127 (2008).

[20] S. Bandyopadhyay, P.O. Boykin, V. Roychowdhury, F. Vatan, Algorithmica 34, 512 (2002).

[21] J. Lawrence, Phys. Rev. A 70, 012303 (2004).

[22] P.K. Aravind, Z. Narufforsch. 58, 85 (2003).

[23] I. D. Ivanovic, J. Phys. A: Math. Gen. 14, 3241 (1981).

[24] W.K. Wootters, B.D Fields, Ann. Phys. 191, 363- 
$381(1989)$

[25] See, for example, the quantum information open problem \#13 in Prof. Werner's homepage: http://www.imaph.tubs.de/qi/problems/13.html

[26] B. G. Englert and Y. Aharonov, Z. Naturforsch. A 56, 16 (2001); B. G. Englert and Y. Aharonov, Phys. Lett. A, 284, 1 (2001).
[27] A. Klappenecher, M. Rotteler, Finite Fields and Aplications, 2948, 137 (2004).

[28] M. Grassl, arXiv:quant-ph/0406175.

[29] P. Horodecki, M. Horodecki, R. Horodecki, Phys. Rev. Lett. 82, 5 (1999). 